\title{
Predicting Annual Equirectangular Panoramic Luminance Maps Using Deep Neural Networks
}

\author{
Yue Liu $^{1}$, Alex Colburn ${ }^{2}$, Mehlika Inanici ${ }^{1}$ \\ ${ }^{1}$ University of Washington, Seattle, USA \\ ${ }^{2}$ Zillow Group, Seattle, USA
}

\begin{abstract}
Annual luminance maps provide meaningful evaluations for occupants' visual comfort, preferences, and perception. However, acquiring luminance maps require labor-intensive and time-consuming simulations or impracticable long-term field measurements. This paper presents a novel method to accelerate annual luminancebased evaluations utilizing a deep neural network (DNN). From a small subset (5\%) of high dynamic range (HDR) imagery, our method can predict annual panoramic luminance maps (with 360-degrees horizontal and 180degrees vertical field of view) within an hour. Unlike the fixed camera viewpoint of perspective or fisheye projections that are commonly used in daylighting evaluations, panoramas allow full degree-of-freedom in camera roll, pitch, and yaw, thus providing a robust source of information for an occupant's visual experience in a given environment. The DNN predicted high-quality panoramas are validated against Radiance RPICT renderings using a series of quantitative and qualitative metrics. With the developed workflow, practitioners and researchers can incorporate long-term luminance-based metrics over multiple view directions into the design and research process without the lengthy computing processes.
\end{abstract}

\section{Introduction}

Design and research practices of daylighting are shifting from illuminance-based metrics towards human centric luminance-based evaluations. Luminance maps capture the subject's field of view and provide millions of values that can be used to evaluate the variabilities in the luminous environment, such as the luminance variations, contrast, positions and intensity of light sources, and background luminance levels. Luminance distribution patterns determine the visual comfort (e.g., discomfort glare), visual appearance and task visibility in a given scene (Wienold and Christoffersen, 2006; Jakubiec and Reinhart, 2012; Suk et al., 2013; Konis, 2014; van den Wymelenberg and Inanici, 2015; Rockcastle et al, 2016).

Meaningful daylighting evaluations require long-term analysis to have a comprehensive understanding of the luminous environment under a wide range of naturally occurring sky and sun conditions. Ideally, daylighting evaluation period should be annual to capture the seasonal variations of daylight, and the time step should be small enough (e.g., 1 hour) to capture the observed short-term daylight variations. However, annual luminance-based simulations require generating a luminance map at each time step of the entire year (approximately 3500-4000 daylight hours), resulting in significant simulation time.

Many accelerating approaches have been developed to make long-term daylighting simulations more efficient and accessible to design professionals. In 1983, a numerical methodology of classic Daylight coefficient (DC) (Tregenza and Waters) approach was developed to accelerate annual daylighting simulations by using an efficienct approximation based on the concept of dividing the celestial hemisphere into discrete sky segments and calculating the sensor's illuminance by accumulating the contribution of each segment. Based on this methodology, multi-phased DC approaches (Laouadi et al., 2008; Ward et al., 2011) have been proposed to improve the simulation accuracy of complex fenestration systems. However, multi-phased DC methodologies are still computationally expensive and require steep learning curves.

Recent accelerating methodologies of long-term daylighting evaluations leverage the developments in modern computing technologies. One solution is to utilize parallel computing to trace multiple primary rays simultaneously on a graphics process unit (GPU) (Jones and Reinhart, 2014). Due to its highly parallel structure, a modern GPU can efficiently process a large block of data, which is not achievable by a general-purpose central processing unit. The second solution is to utilize machine learning to create predictive models from observed data, bypassing the expensive computations involved with physcially based modelling systems. Researchers have investigated learning-based models for illuminance predictions (Kazanasmaz et al., 2009; Zhou and Liu, 2015; Navada et al., 2016; Ahmad et al., 2017). Though inspiring, illuminance predictions are less complicated compared with luminance predictions due to lower resolutions of calculation points and lack of dependency on view directions. As a result, annual illuminance predictions can be achieved through standard simulation practices without significant simulation time.

The objective of this study is to explore the use of machine learning, particularly deep neural networks, to accelerate long-term per-pixel luminance predictions of 
indoor scenes. Statistical methods have been investigated for per-pixel luminance predictions (Inanici, 2013). They succeed in predicting images under overcast skies but require further improvements for sunny sky conditions. Our machine learning methodology is built upon the algorithm developed by Liu et al. (2018), which demonstrated that DNN models can solve complicated rendering problems by accurately predicting the annual luminance maps of a fixed perspective view from $5 \%$ of annual renderings. We have improved Liu et al.'s model through multiple algorithmic refinements, which will be discussed in the following sections. The results show increased accuracy for luminance predictions under various sky conditions. We have utilized $360^{\circ}$ panoramas as opposed to single fixed perspective views. There is an increasing demand for panoramic luminance maps as recent studies in luminance-based evaluations integrate subject's visual experience over multiple viewing directions (Jakubiec and Reinhart, 2012; Rockcastle et al., 2017). However, generating panoramic luminance maps is even more time-consuming than single fixed-view perspectives.

This study provides an end-to-end workflow that regresses annual HDR panoramas from sparse samples using a DNN framework. The DNN approach accelerates the generation of panoramic luminance maps and only requires a few seconds of computing time during evaluation. The accuracy, applicability, and usefulness of the proposed DNN model is examined using a series of quantitative and qualitative metrics. We share a public dataset of high-quality annual HDR panoramic luminance maps to enable reproducibility and future explorations (https://github.com/yueAUW/neural-daylighting.git).

\section{Learning from the HDR Panoramas}

\section{Artificial Neural Network}

An artificial neural network (ANN) (McCulloch and Pitts, 1943) is a powerful machine learning technique for modeling complex systems. ANNs are composed of neuron-like connected computing nodes, organized into hierarchic layers from input layers, hidden layers, to output layers. Each pair of the connected nodes are assigned a weight parameter which controls the degree of influence a node has on its connected pair. An ANN finds the relationships between inputs and outputs through a learning process. The learning process involves multiple steps: 1) input data passes through the input layers towards the output layers; 2) at the output layer, the predicted results are calculated and compared with the ground truth using an error metric termed a loss function; 3 ) the weight parameters are updated according to the calculated errors; 4) the previous steps are repeated several times (epochs) until the error between the prediction and ground truth is minimized. After the model is trained, it can be used for predictions given new input data. A DNN consists of more hidden layers between the input and output layers than a shallow ANN and can learn more complicated non-linear relationships. In this work, we use a DNN to learn the light transport model which represents the relationship between input lighting conditions and output luminance maps.

\section{Light Transport Model}

The light transport model describes how light travels in a scene. In traditional physically-based renderings, a light transport model is computed using the complete scene information including the light sources, geometries, and materials. In this study, the light transport model is inferred from sparse samples of luminance maps generated under various illumination. We use a DNN to estimate the light transport model $(M)$ through learning the non-linear relationship between Illumination conditions (input $I$ ) and the corresponding pixel luminance values (output $L$ ). The estimated light transport model $(M)$ allows the scene to be rendered under novel lighting conditions. Using this method, generating annual luminance maps becomes a three-step process that involves: 1) acquiring sparse samples of luminance maps; 2) estimating the light transport model from the sparse samples using the DNN, and; 3) predicting annual luminance maps using the estimated light transport model. The total calculation time is significantly reduced compared with the traditional annual simulation method. Instead of rendering luminance maps for the entire year, we only need to render a small set of them.

The illumination condition (input $I$ ) is parameterized with the following features: 1) the pixel location defined by the $\mathrm{x}$ and $\mathrm{y}$ coordinates $(p x, p y) ;(2)$ the sun location defined by sun altitude and azimuth angles $(a l, a z) ; 3)$ the sky condition defined by direct and diffuse irradiances (dir, dif), which are main input parameters to generate sky luminance maps using the Perez sky model (Perez et al. $1993)$; 4) the average luminance value for an image pixel (avg), to improve convergence (Ren et al., 2015); 5) the pixel luminance of the sun patches (sunpatch) which aids the neural network in reconstructing sharp shadows and sun penetrations (Liu et al., 2018). Our studies show that augmenting the input by adding Perez sky luminance maps (skymap) improves the accuracy of the predicted luminance maps. In comparison to direct and diffuse irradiance parameters, panoramic Perez sky maps provide spatial information of the sky luminance distributions. Both sun patches and sky maps can be simulated quickly, thus have little impact on the simulation efficiency. The input feature vector includes 9 parameters $I=(p x, p y, a l$, az, dir, dif, avg, sunpatch, skymap). The output vector $L$ is the pixel luminance under the given lighting condition.

To develop the methodology under controlled settings, we train and evaluate the neural networks with highquality HDR images and demonstrate that our method can reconstruct accurate luminance maps. Figure 1 gives an overview of the proposed methodology. The workflow starts with the sample data generation process using the Radiance rendering engine (Ward, 1994). For each daylighting condition, the proposed system uses Radiance to generate a set of three HDR panoramic images consisting of high-quality interior maps, sky maps, and low-quality sun patches. The interior maps are used as ground truth, and the sky maps and sun patches are input 


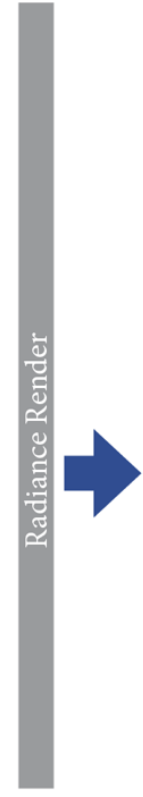

a) Rendering

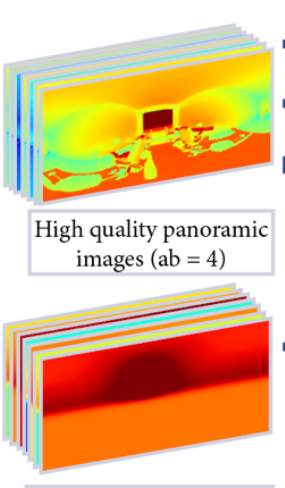

Panoramic sky maps

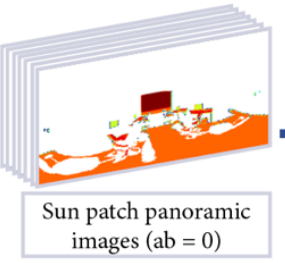

b) Data Generation

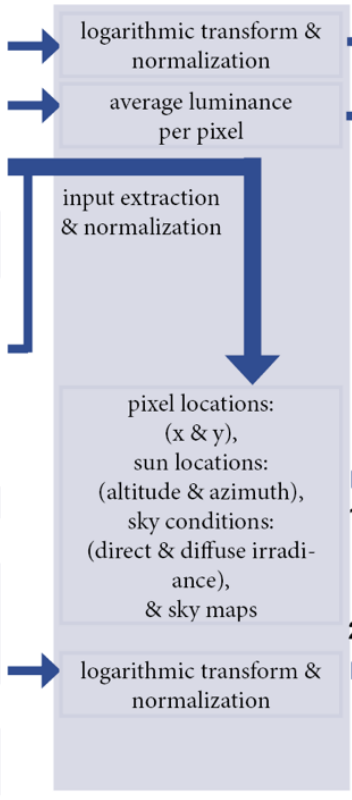

c) Preprocessing

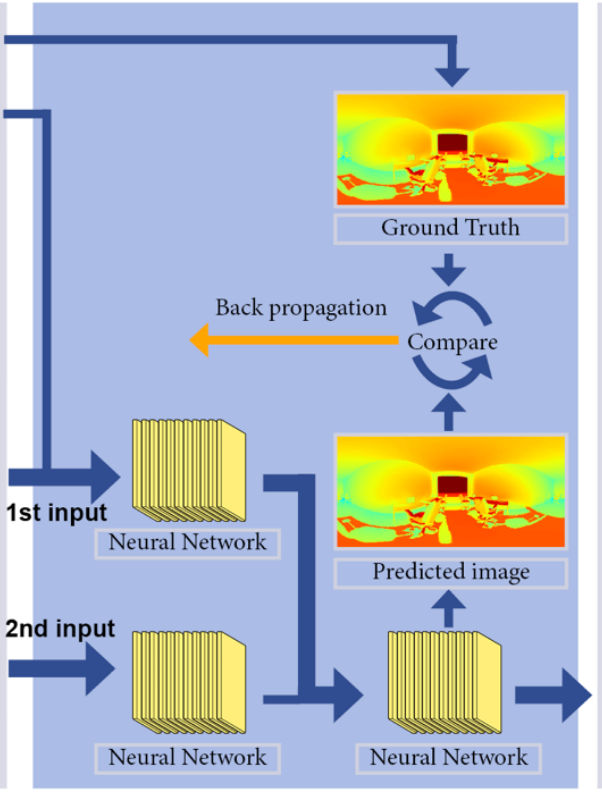

d) Training

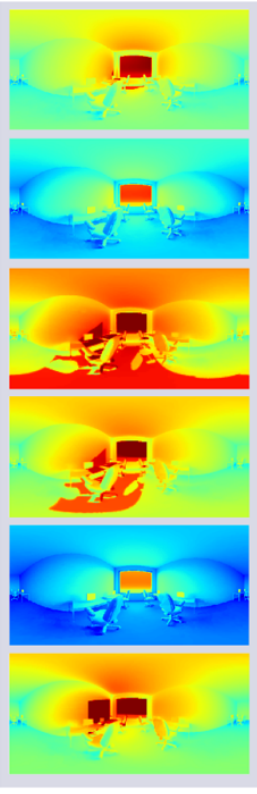

e) Prediction

Figure 1: Overview of the workflow: a) A sparse data set is rendered using the Radiance simulation engine. b) Each data point includes three panoramas consisting of interior maps, sky maps, and sun patches. The interior maps are used as ground truth for the DNN training process, while the sky maps and the sun patches are used as the input to the DNN. c) All images are pre-processed and normalized. The sun patches and simulation parameters are combined to create the input for the DNN. d) The network is trained to predict the interior maps. e) The trained network can then be used to generate interior maps from novel sky maps, sun patches, and simulation parameters. Further long-term luminancebased analysis (e.g., glare and spatial contrast analysis) can be performed using these predicted interior panoramas.

to the neural network training. After the training is finished, the light transport model is estimated and can be used to predict luminance maps under new lighting conditions.

\section{Data Generation}

The test room for the simulation is set to be an open plan office with basic furniture arrangement and a south facing side window, located in Seattle $\left(47.6^{\circ} \mathrm{N}, 122.3^{\circ} \mathrm{W}\right)$ (Figure 2). The dimension of the room is $6 \mathrm{~m}$ (width) by $14 \mathrm{~m}$ (depth) and $4.5 \mathrm{~m}$ (height). The camera is set to be in the front center of the room at eye level $(1.6 \mathrm{~m}$ from the floor) to correspond to a standing human's perspective. The materials for walls, ceiling, floors, and outdoor ground have reflectance values of $50 \%, 80 \%, 20 \%$, and $20 \%$ respectively. The interior maps are rendered with 4 ambience bounces (-ab 4) and sun patches are rendered with 0 ambient bounce $(-\mathrm{ab} 0)$; they share the other

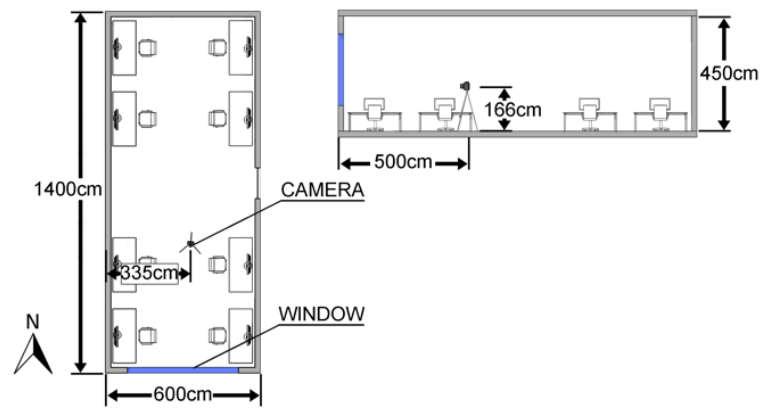

Figure 2: Plan and section of the test scene used in Radiance simulations
RPICT rendering parameters (-ps 2 -pt .05 -pj .9 -dj .7 -ds .15 - dt .05 -dc . 75 -dr 3 -st .15 -aa .1 -ar 512 -ad 2048 -as 1024 -lr 8 -lw .005). Sky maps are generated with the same rendering parameters as the interior maps. We generate all data with Perez all-weather sky model with inputs of direct and diffuse irradiances extracted from EnergyPlus weather file. All images are rendered as equirectangular (spherical) panoramas from a single viewpoint (Figure 3). The panoramas are rendered with a pixel resolution of $1840 \times 920$ and downsized to $460 \times$ 230 using Radiance pfilt for anti-aliasing. All sets of images are generated in 1-hour intervals from sunrise to sunset for the entire year, resulting in 4379 data samples.

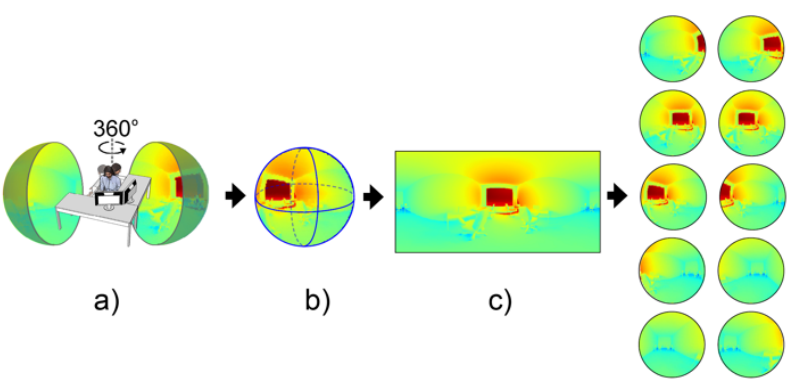

d)

Figure 3: a) and b) Full $360^{\circ}$ field of view models an occupant's visual experience and provides more complete evaluations than a single fixed perspective view; Radiance generated equirectangular panoramas are used as (c) inputs to the DNN and then converted to fisheye images (d) for visual comfort analysis. 
Generating such a large dataset enables developing and testing models. When applying the workflow into practice, the user only needs to provide a small amount of HDR images.

\section{Data Pre-processing}

Data pre-processing is an important step. Initially, all features are normalized to the range of 0 to 1 . Feature normalization tends to improve the training process by insuring that all features are in the same scale. Due to the nature of high dynamic range renderings, the luminance values of the scene can span several orders of magnitude (e.g., the brightest part of the solar corona compared to the shadows in the room), and are not evenly distributed throughout the range. In our database, the range of the ground truth is $10^{0}-10^{+8.3} \mathrm{~cd} / \mathrm{m}^{2}$. The luminance of the overhead sun, $1.6 \times 10^{+9}$ (Karandikar, 1955), is used to scale luminance values. After the feature scaling, the majority of these values are very low. This leads to underestimation of the lower range of luminance values and results in poor prediction performance. Several processing steps are performed on the luminance intensities to better distribute the values throughout the range. First, a $\log _{10}$ transform is applied to spread the range of luminance more evenly, and the maps are normalized to the range of $[0,1]$. Then a gamma correction of 1.5 is performed to shift the intensity distribution towards a mean value of $1 / 2$. Our experiments show that the model performance is greatly improved after the pre-processing. When the training is completed, a reverse transform is applied to reconstruct the high dynamic range luminance maps.

\section{Network Architecture}

The machine learning model in this study is built upon the model developed by Liu et al. (2018). In their study, a Convolutional Neural Network (CNN) model is used to regress the light transport model from the training input. However, this model has difficulties learning fine details under different lighting conditions. Since training the CNN requires the complete panorama input $(460 \times 230 \times$ 9), small batch sizes are required during training due to GPU memory limitations. In other words, only a few lighting conditions are presented to the network in each batch step during training. The limited amount of data in the small batch does not well represent the overall data distribution of the entire model, and the training does not converge as well as it could if each batch better represented the data distribution. To overcome this limitation, we use a multi-input deep dense network model architecture. The input data at each training step is sampled from all pixels and various lighting conditions in the entire training set. Experiments show that the updated model and batch sampling strategy greatly reduces the prediction error.

Our network architecture divides the 9 input features into two separate vectors as input to a two-input deep network consisting of fully connected layers with ReLU activations. The first input branch, which encodes $p x, p y$, $a l, a z$, dir, dif, avg, and skymap, consists of four fully connected layers, each with 600 nodes. The second input branch, which encodes sunpatch, consists of a single 400 nodes fully connected layer. The first input branch encodes parameters describing global illumination while the second input branch encodes direct sun illumination. The two branches are concatenated to form a 1000 node layer, which is followed by a fully connected layer of 600 nodes. The output layer is a single fully connected node that predicts luminance values. During training, the differences between predicted and ground truth luminance values are calculated using a loss function, and the model weights are updated accordingly.

The loss function in this study is composed of two functions: $\operatorname{Loss}(y, t)=\mathcal{L}_{M S E}(y, t)+\lambda \times \mathcal{L}_{R E R}(y, t)$, where $\mathrm{y}$ is the network prediction, $\mathrm{t}$ is the ground truth, and $\lambda$ controls the relative weights between two functions. The first function calculates the L2 distance (MSE) between the predicted and ground truth luminance $\mathcal{L}_{M S E}(y, t)=\frac{1}{n} \sum_{i=1}^{n} \omega_{i}\left(y_{i}-t_{i}\right)^{2}$, where $\omega_{i}$ is the solid angle for pixel i. Solid angle weight $\omega_{i}$ is added so that each pixel is appropriately weighted for spherical panoramic solid angle projection (Figure 4). A second loss of $\mathcal{L}_{R E R}(y, t)=\omega_{i} \sqrt{\frac{\sum_{i}\left(y_{i}-t_{i}\right)^{2}}{\sum_{i} t_{i}{ }^{2}}}$ is added to improve the model performance with a weight $\lambda$ of 10 .
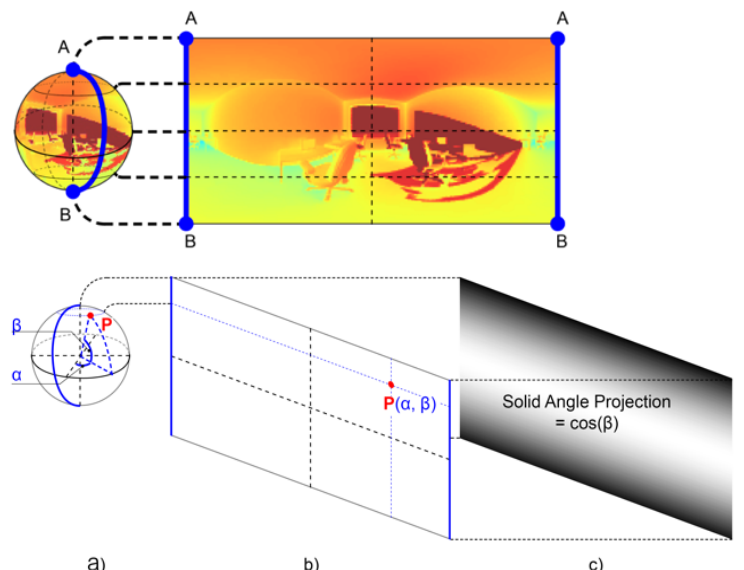

Figure 4: (Top) The equirectangular (spherical) panorama is mapped from a unit sphere. The north pole $(A)$ and south pole $(B)$ on the sphere become the top $(A A)$ and bottom $(B B)$ edges of the corresponding panorama. (Bottom) The image shows the solid-angle projection of each pixel in the panorama.

\section{Training Details}

The generated images are divided into training, validation and test sets. The model learns to approximate the light transport model from the data in the training set and evaluates its performance with the validation set during training. After the model is trained, its performance is evaluated using the test set. Note that there is not an overlap between the test/validation dataset and the training set. Hence, all tests are performed for lighting conditions that have not been seen by the network before. K-means clustering (i.e., a clustering method which partitions the data into $k$ relatively equal-sized groups) is utilized to select the training samples evenly distributed 
over the light domains (Figure 5) in order to cover various sun positions and sky conditions.
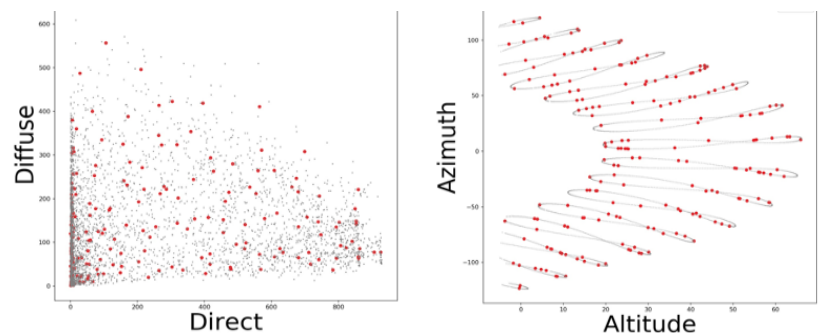

Figure 5: The K-means method is utilized to make the selected training samples well-distributed over the 4dimensional light domain visualized in two 2dimensional plots: (Left) distribution plot over sky condition dimensions (direct and diffuse irradiances), and (Right) over sun position dimensions (azimuth and altitude angle). Red dots represent the selected training samples while grey dots represent all data points.

We use the ADAM optimizer (Kingma and $\mathrm{Ba}, 2014$ ) with an initial learning rate of $10^{-3}$, reducing the learning rate by a factor of 2 once learning stagnates, and a minibatch size of $6 \times \mathrm{N}$, where $\mathrm{N}=$ width $\times$ height $\times 8$ is the total number of input parameters in one image. The minibatch size of 6 is selected so it fills the 11GB memory of the GPU. The model is trained for 30 epochs (which we find sufficient) or until the validation loss reaches a minimum threshold $\left(10^{-10}\right)$. Training takes roughly 30 minutes on a Nvidia GeForce 1080Ti GPU. At test time, predicting a high-quality panoramic luminance map only takes $1 / 10$ second.

\section{Results and Analysis}

The neural network model is trained with 200 samples ( $5 \%$ of 4379 images). The performance of the trained model is then evaluated on the test set of 500 randomly selected lighting conditions, which have not been seen by the network before. The predicted panoramas are compared with the panoramas rendered by Radiance using per-pixel errors, falsecolor analysis with image subtraction operations, and human-centric metrics (visual comfort and perceptual metrics).

The first set of metrics are per-pixel errors measured by $\mathcal{L}_{M S E}$ and $\mathcal{L}_{R E R}$ used in the loss function. The errors are solid projected angle weighted and calculated with logarithmic $\left(\log _{10}\right)$ luminance values to correlate with the human visual system (Fechner, 1966). Note the range of ground truth intensities is $[0,8.3]$. Our network succeeded in achieving a $\log _{10} \mathcal{L}_{M S E}$ of $9.85 \mathrm{e}-03$ and a $\mathcal{L}_{R E R}$ of $4.93 \mathrm{e}-02$ on the test set.

Per-pixel errors do not necessarily prompt significant differences in architectural design evaluations. For

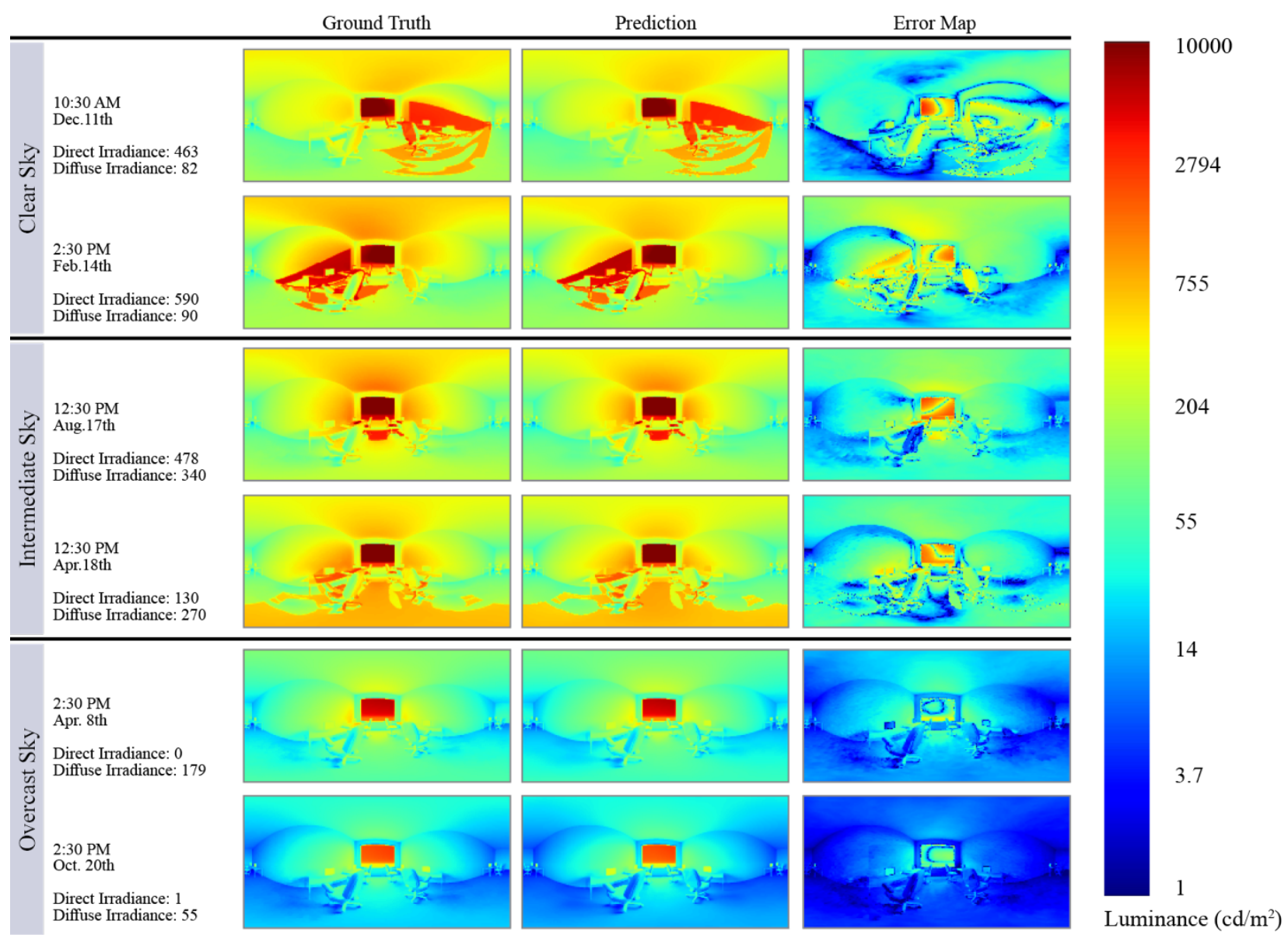

Figure 6: Six exemplary test cases with different sky types are displayed in falsecolor with a logarithmic scale. 
example, if the sun is estimated to be located a pixel off its ground truth location, a significant $\boldsymbol{L}_{M S E}$ and $\boldsymbol{L}_{\boldsymbol{R E R}}$ error will incur but will hardly lead to a different design decision. Here, the per-pixel errors are provided as benchmarks for future studies. Metrics that are commonly used by design professionals, such as falsecolor images, daylight glare probability (DGP) (Wienold and Christoffersen, 2006), and RAMMG (Rizzi et al., 2004) are utilized to evaluate the results.

\section{Falsecolor Image Comparison}

Falsecolor images are commonly used to study the luminance distributions and the resulting visual effect, comfort and performance. In Figure 6, we show the comparison results in falsecolor with a logarithmic scale, with error maps illustrating the absolute difference between ground truth and predicted luminance maps. We select six representative samples under different sky conditions (clear, intermediate, and overcast) from the test set. The results show that: 1) Our predicted luminance panoramas (generated from 5\% sparse samples) are visually imperceptible from ground truth results in falsecolor images without the help of error maps. This implies that the proposed method will lead to the same design decisions as conventional rendering methods, with orders of magnitudes less of calculation time. 2) Among all sky conditions, images of the clear sky with high direct irradiance values are most challenging to predict with the highest error. This is mostly due to the higher luminance ranges of these images than those of the overcast sky. 3) The error maps show that higher errors in each case occur in the window area with the highest ground truth luminance. Overall, we obtain high-quality results illustrating the ability of our network to predict panoramic luminance maps under novel lighting conditions. Figure 7 shows the comparison between our model and the one proposed by Liu et al. (2018). Our model can handle higher luminance range $\left(10^{8}\right)$ and generate images with clearer boundaries and finer details. The applicable

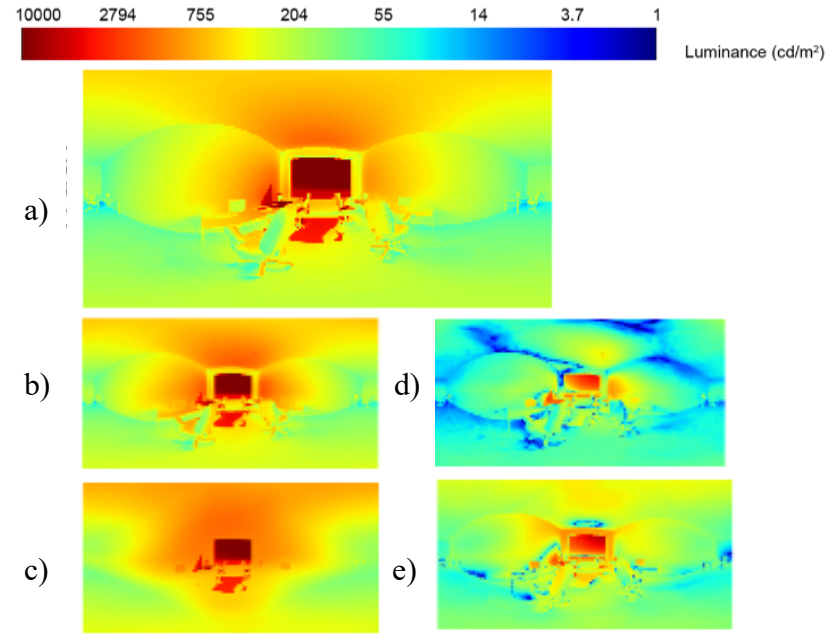

Figure 7: (a) the ground truth luminance map, luminance map predictions of our model (b) is compared to that of (c) Liu et al. (2018). (b) has clearer boundaries and finer details, indicating higher accuracy on the lower range values. (d) and (e) are errors maps for (b) and (c).

luminance range of Liu et al.'s model is $0-3 \times 10^{4}$ while our panorama data is $0-10^{8}$.

\section{DGP}

DGP is a visual comfort indicator that describes the subjective magnitude of discomfort glare with high values illustrating the higher percentage of the population who would perceive the scene as glary (Wienold and Christoffersen, 2006). The output of the DGP model is given on a scale of four ordinal values, ranging from imperceptible $(<0.35)$, perceptible $(0.35$ to 0.40$)$, disturbing ( 0.40 to 0.45$)$, to intolerable $(>0.45)$ glare.

For DGP analysis, each panoramic luminance map in the test set is converted to 10 equidistant fisheye images over 360 degrees of rotation in the y-axis using 36-degree increments (Figure 3). DGP is then applied to both the predicted and ground truth fisheye images. Figure 8 shows the per-view-angle DGP comparison of an

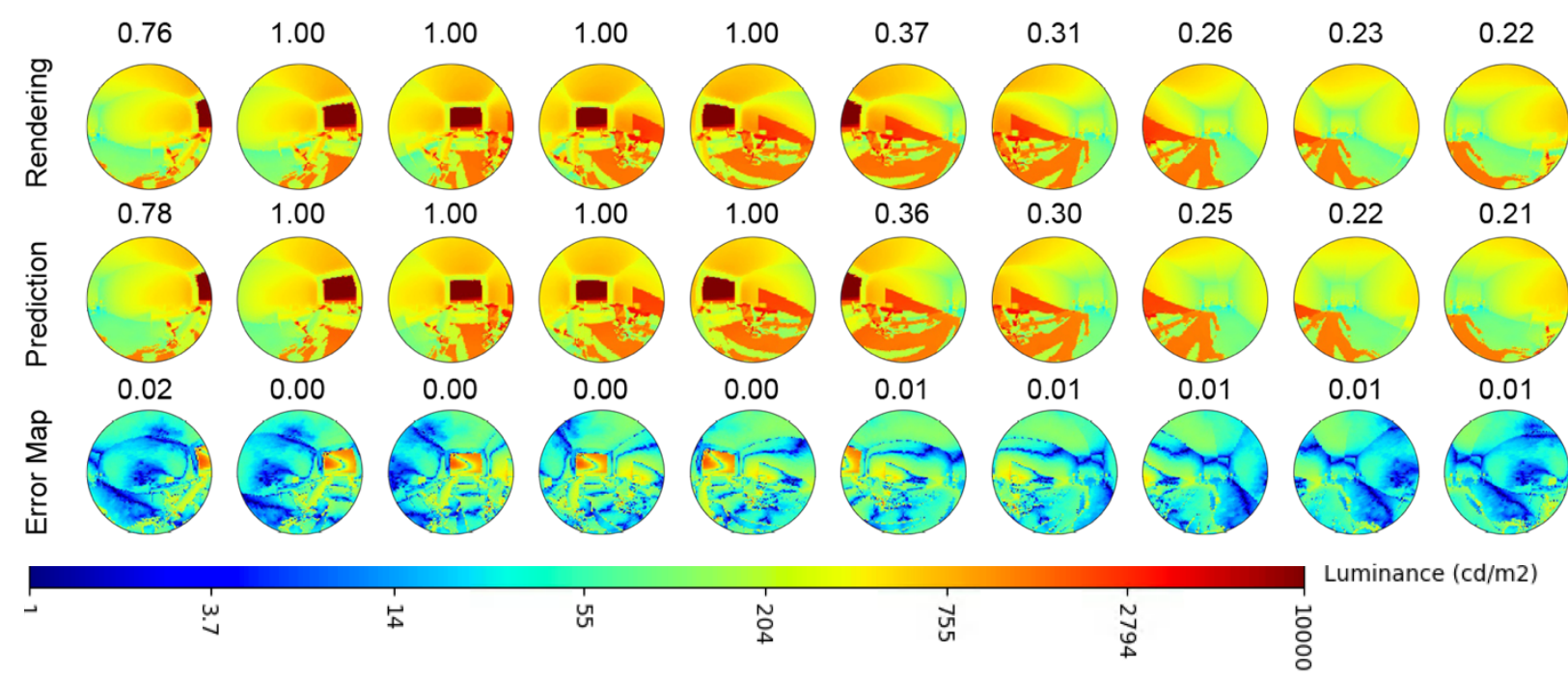

Figure 8: Comparison of Radiance rendered and DNN predicted fisheye images over a 360 degree view in 36 degree increments. The ground truth and predicted fisheye images are labeled with the DGP value. The error maps are labeled with absolute difference in DGP values. All images are shown in false color with a logarithmic scale. 
exemplary test lighting condition (January $21^{\text {st }} 11: 30 \mathrm{am}$, direct and diffuse irradiances of 553 and $87 \mathrm{~W} / \mathrm{m}^{2}$ ), with error maps illustrating the absolute differences. The result illustrates that 1) a subject's visual comfort level can change vastly across various view directions of the same scene depending on the shift of the sun location into and out of the field of view, 2) the neural network model accurately predicts the overall luminance environment, with the predicted DGP values from fisheye images closely matching those of ground truth ones in all directions, and 3) the more significant errors occur in the window region.

Figure 9 shows the comparison scatter plot of the DGP values for all of the test lighting conditions. The analysis shows a strong agreement overall (with an $\mathrm{r}^{2}$ of 0.992), but exhibits a bias with the predicted model underestimating DGPs at higher ranges. Further investigation shows the high errors occur during winter at midday, when sun direct appears in the field of view through the south-facing window (Figure 10). The model has difficulties in estimating the sun luminance $\left(10^{+9}\right.$ $\mathrm{cd} / \mathrm{m}^{2}$ ) which is multiple orders of magnitudes higher than the rest part in the scene. The failure cases are negligible as 1) these cases only account for about $1 \%$ of the total tests cases; 2) the predictions still fall into the same zone (intolerable) as the ground truth DGPs, and 3) the situation is avoidable in the real world when people have an ability to change their view directions and positions to avoid a direct view of the sun. For scenes with actual DGP in the imperceptible, perceptible and disturbing zone, our method produced very little variation.
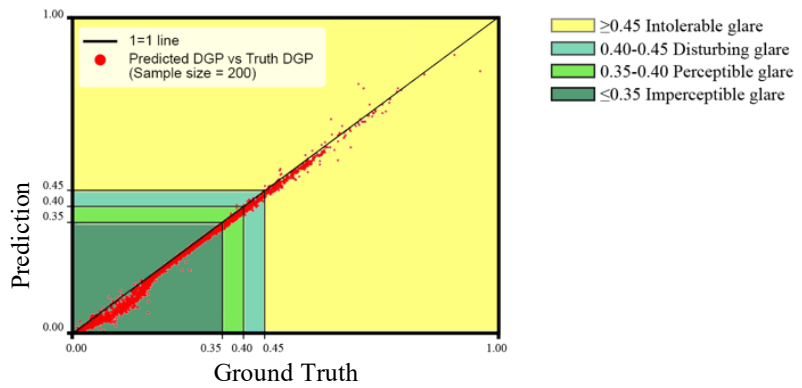

Figure 9: Scatterplot comparison of DGP values calculated using ground truth and predicted images.
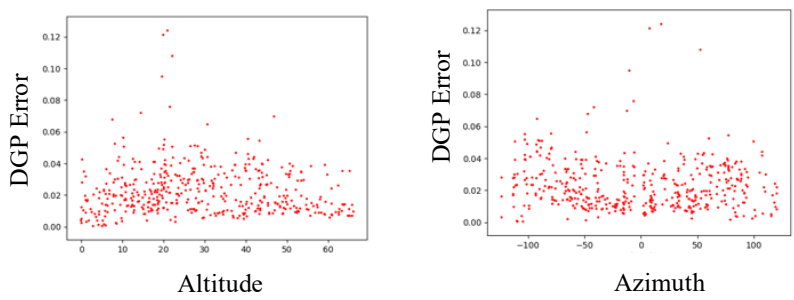

Figure 10: Scatter plots of DGP prediction errors (differences between predictions and ground truth) and the sun altitude (left) and azimuth (right). The max errors occur at sun altitude and azimuth of roughly 20 and $0^{\circ}$, respectively, when sun directly appears in the field of view through the south window during winter at midday.

\section{RAMMG}

RAMMG is a contrast measure of images and is utilized as a perceptual predictor of contrast-based visual characteristics of daylight (Rockcastle et al., 2016). RAMMG performs a pyramid subsampling of the image to various levels (at each level, the resolution is reduced to half of the previous level) and combines the average contrast calculated at each level. RAMMG is applied to both the predicted panoramas and Radiance rendered panoramas in the test set. Subsampling of five levels is used taking into consideration the image resolution (460 by 230). Figure 11 illustrates the correlation between the predicted and ground truth RAMMGs; it shows a strong agreement $\left(r^{2}\right.$ of 0.978$)$ with a few errors at higher ranges when the sun directly appears through the window.
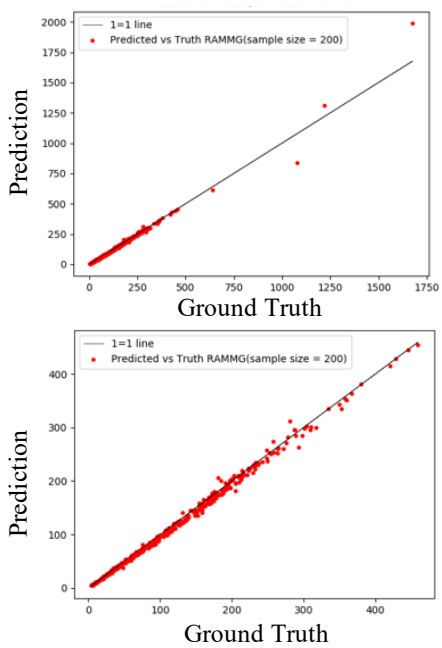

Figure 11: Comparison of RAMMG values calculated using ground truth and predicted images: (top) original data, and (bottom) zoomed in data.

\section{Conclusion}

In this paper, we have developed a robust workflow to perform annual luminance-based evaluations that are orders of magnitudes faster than the conventional simulation methods. Our method leverages a deep neural network to learn the non-linear light transport model from sparse samples $(5 \%)$ of HDR imagery and predict annual panoramic luminance maps under new lighting conditions. The predicted panoramic luminance maps can be transformed into fisheye projections for any view direction. We demonstrated the accuracy of the method through comparisons with Radiance RPICT renderings using per-pixel error metrics, visual comfort, and contrast metrics.

Architects, lighting designers, and researchers can effectively use the workflow to perform multi-view annual lighting analyses. Making annual luminancebased simulations more accessible will enable further development of luminance-based metrics for daylighting studies and foster wider usage of human-centric evaluations in the design processes. This methodology 
can be applied for generating annual luminance maps utilizing HDR photographs of existing environments. As a future work, we plan to shorten the required data collection period to facilitate the feasible gathering of HDR photography-based training sets. We also plan to compare the model performance and accuracy against multi-phased daylight coefficient methods.

\section{References}

Ahmad, M.W., Hippolyte, J., Mourshed M., Rezgui, Y. (2017). Random Forests and Artificial Neural Network for Predicting Daylight Illuminance and Energy Consumption. Proceedings of BS2017. San Francisco (USA).

Fechner, G.T., Howes, D. H., Boring, E. G. (1966) Elements of psychophysics. volume 1. Translated by Adler, H.E: Holt, Rinehart and Winston.

Inanici, M. (2013) Dynamic Daylighting Simulations from Static High Dynamic Range Imagery Using Extrapolation and Daylight Coefficient Methodologies. Proceedings of BS2013. Chambéry.

Jakubiec, J.A., Reinhart, C. (2012) The 'adaptive zone' A concept for assessing glare throughout daylit spaces. Lighting Research and Technology 44, 149-170.

Jones, N., Reinhart, C. (2014). Irradiance Caching for Global Illumination Calculation on Graphics Hardware. Ashrae/Simbuild, 111-20.

Karandikar, R.V. (1955). Luminance of the Sun. J. Opt. Soc. Am. 45, 483-488.

Kazanasmaz, T., Gunaydin, M., Binol, S. (2009) Artificial Neural Networks to Predict Daylight Illuminance in Office Buildings. Building and Environment 44 (8): 1751-57.

Kingma, D., and Ba, J. (2014) Adam: A method for stochastic optimization. Proceedings of the 3rd International Conference on Learning Representations.

Konis, K. (2014) Predicting Visual Comfort in Side-Lit Open-Plan Core Zones: Results of a Field Study Pairing High Dynamic Range Images with Subjective Responses. Energy and Buildings 77.: 67-79.

Laouadi, A., Reinhart, C.F., and Bourgeois, D. (2008) Efficient Calculation of Daylight Coefficients for Rooms with Dissimilar Complex Fenestration Systems. Journal of Building Performance Simulation 1: 3-15.

Liu Y, Colburn A, and Inanici M. (2018) Computing Long-term Daylighting Simulations from High Dynamic Range Imagery Using Deep Neural Networks. 2018 SimBuild, Chicago (USA).

McCulloch, W.S. and Pitts, W. (1943) A Logical Calculus of the Ideas Immanent in Nervous Activity. The Bulletin of Mathematical Biophysics 5 (4): 115-33.
Navada, S.G., Adiga, C.S., and Kini, S.G. (2016) Prediction of Daylight Availability for Visual Comfort. International Journal of Applied Engineering Research 11 (7): 4711-17.

Perez, R., Seals, R., and Michalsky, J. (1993) All-Weather Model for Sky Luminance Distribution-Preliminary Configuration and Validation. Solar Energy 51: 423.

Ren, P., Dong, Y., Lin, S., Tong, X. and Guo, B. (2015) Image Based Relighting Using Neural Networks. ACM Transactions on Graphics 34 (4), No. 111.

Rizzi, A., Algeri, T., Medeghini, G., and Marini, D. (2004) A proposal for Contrast Measure in Digital Images. 2. European Conference on Color in Graphics, Imaging and Vision 187-192.

Rockcastle, S., Ámundadóttir, ML., and Andersen, M. (2016) Contrast measures for predicting perceptual effects of daylight in architectural renderings. Lighting Research and Technology 49(7), 882-903.

Rockcastle, S., Chamilothori, K., Andersen, M. (2017) An Experiment in Virtual Reality to Measure Daylight-Driven Interest in Rendered Architectural Scenes. Proceedings of BS2017. San Francisco (USA).

Suk, J.Y., Schiler, M., and Kensek, K. (2013) Development of New Daylight Glare Analysis Methodology Using Absolute Glare Factor and Relative Glare Factor. Energy and Buildings (64):113-22.

Tregenza, P.R. and Waters, I.M. (1983). Daylight Coefficients. Lighting Research and Technology 15(2): 65-71.

van den Wymelenberg, K. and Inanici, M. (2015) Evaluating a New Suite of Luminance-Based Design Metrics for Predicting Human Visual Comfort in Offices with Daylight. Leukos 2724 (October): 1-26.

Ward, G., Mistrick, R., Lee, E.S., Mcneil, A., Jonsson, J. (2011) Simulating the Daylight Performance of Complex Fenestration Systems Using Bidirectional Scattering Distribution Functions within Radiance January. Leukos, 7(4).

Ward, G., (1994). The RADIANCE Lighting Simulation and Rendering System, Computer Graphics (July): 459-72.

Wienold, J. and Christoffersen, J. (2006). Evaluation methods and development of a new glare prediction model for daylight environments with the use of CCD cameras. Energy and Buildings 38(7), 743-757.

Zhou, S. and Liu. D. (2015). Prediction of Daylighting and Energy Performance Using Artificial Neural Network and Support Vector Machine. American Journal of Civil Engineering and Architecture 3, 1-8. 